\title{
A real-world example of using personal and public involvement to develop a healthy eating and physical activity intervention for pregnant women
}

\author{
J. McClelland ${ }^{1}$, L. McGowan ${ }^{1}$, D. Gallagher ${ }^{1}$, S.E. Moore ${ }^{1}$, R.J. Beeken ${ }^{2}$, C.R. Cardwell ${ }^{1}$, \\ H. Croker ${ }^{3}$, K.A. Eastwood ${ }^{1,4}$, C. O’Neill ${ }^{1}$, R.F. O’Neill ${ }^{1}$, J.V. Woodside ${ }^{1}$ and M.C. McKinley ${ }^{1}$ \\ ${ }^{1}$ Centre for Public Health, School of Medicine, Dentistry and Biomedical Sciences, Queen's University Belfast, Belfast, \\ $U K$, \\ ${ }^{2}$ Leeds Institute of Health Sciences, School of Medicine, University of Leeds, Leeds, UK, \\ ${ }^{3}$ World Cancer Research Fund, London, UK and \\ ${ }^{4}$ St Michael's Hospital, University Hospitals Bristol and Weston NHS Foundation Trust, Southwell Street, Bristol, UK
}

This abstract was awarded the student prize.

There is limited support available to women with overweight and obesity to manage weight during pregnancy. A brief leaflet-based intervention, "Ten Top Tips for a Healthy Weight" (10TT), successfully used habit-formation to facilitate behaviour change for weight-management in adults ${ }^{(1)}$. Personal and public involvement in research (PPI) is important in the development of complex behavioural interventions to ensure they are relevant and acceptable to the target population ${ }^{(2)}$. This PPI activity aimed to adapt and refine the 10TT intervention to be suitable for pregnancy.

PPI representatives from Northern Ireland $(n=9)$ and Republic of Ireland $(n=1)$ became involved after seeing poster advertisements in antenatal care settings, letters (sent to previous participants in a postpartum weight-management study) and via word-of-mouth. They engaged in Trial Steering Committee meetings, participated in role-plays in intervention training for midwives, provided feedback on intervention materials (leaflet, logbook, app and library of text messages) and reviewed participant-facing documents (questionnaires and information sheets). The research team (nutritionists and psychologists) reviewed the evidence base relating to diet and physical activity guidelines during pregnancy and created the initial drafts of materials. Initially, PPI reviewed the content and tone of materials and following revisions, reviewed the layout. Feedback was completed electronically or on hard-copies, depending on PPI preference. PPI also rated 12 common terms to describe weight (Likert scale: $1=$ strongly dislike to $5=$ strongly like).

PPI reps $(n=6)$ provided feedback from June 2019-September 2020. PPI reps chose the study name 'Healthy Habits in Pregnancy and Beyond (HHIPBe)'. Overall, feedback on the leaflet (PPI $n=6)$, logbook $($ PPI $n=5)$, app $($ PPI $n=3)$ and text messages $($ PPI $n=$ 6) was positive. HHIPBe focused on weight-management instead of weight-loss and included pregnancy-relevant contexts, such as morning sickness and cravings. Tone was modified to be more empathetic and informal. Queries that arose during the PPI process were incorporated into the 'frequently asked questions' section such as, "does green tea contain caffeine?". Preferred terminology to describe weight ('excess weight' (mean = 4.3)) was used throughout. The app was user-tested and a tutorial with instructions was subsequently created. Additional 'handy hints' on portion sizes were included in the library of text messages. PPI reps ensured language used throughout the materials was appropriate and user-friendly.

The original 10TT has been adapted via PPI to be suitable for pregnancy. PPI was integral to the development of every aspect of the intervention, providing invaluable feedback and perspectives and greatly improving the intervention materials. The acceptability of the resulting materials is currently being tested in a feasibility study.

\section{Acknowledgements}

We thank the PPI representatives for their continued input. The CHITIN project has received €8.8m from the EU's INTERREG VA Programme, which is managed by the Special EU Programmes Body (SEUPB).

\section{References}

1. Beeken RJ, Leurent B, Vickerstaff V, et al. (2017) Int J Obes 41, 246-54.

2. INVOLVE (2012) Briefing notes for researchers: involving the public in NHS, public health and social care research. INVOLVE, Eastleigh. [Available at: https://www.invo.org.uk/wp-content/uploads/2012/04/INVOLVEBriefingNotesApr2012.pdf] 